\title{
BMJ Open Rate of condom use among sexually active adolescents: a nationwide cross-sectional study in Taiwan from 2012 to 2016
}

\author{
Wing Hin Stanford Siu (D) , ${ }^{1}$ Pei-Ru Li (D) , ${ }^{2}$ Lai-Chu See (D) ${ }^{2,3,4}$
}

To cite: Siu WHS, Li P-R, See LC. Rate of condom use among sexually active adolescents: a nationwide cross-sectional study in Taiwan from 2012 to 2016. BMJ Open 2021;11:e047727. doi:10.1136/ bmjopen-2020-047727

- Prepublication history and additional supplemental material for this paper are available online. To view these files, please visit the journal online (http://dx.doi.org/10.1136/ bmjopen-2020-047727).

Received 07 December 2020 Accepted 22 June 2021

\section{Check for updates}

(c) Author(s) (or their employer(s)) 2021. Re-use permitted under CC BY-NC. No commercial re-use. See rights and permissions. Published by BMJ.

${ }^{1}$ School of Medicine, College of Medicine, Chang Gung University, Taoyuan City, Taiwan ${ }^{2}$ Department of Public Health, College of Medicine, Chang Gung University, Taoyuan City, Taiwan

${ }^{3}$ Division of Rheumatology, Allergy and Immunology, Department of Internal Medicine, Chang Gung Memorial Hospital at Linkou, Taoyuan City, Taiwan ${ }^{4}$ Biostatistics Core Laboratory, Molecular Medicine Research Center, Chang Gung University, Taoyuan City, Taiwan

Correspondence to

Prof Lai-Chu See;

lichu@mail.cgu.edu.tw

\section{ABSTRACT}

Objective Adolescents' sexual behaviours are associated with sexually transmitted infections and unwanted pregnancies. This study aimed to estimate the sexual intercourse and condom use rates at first and last sex among Taiwanese adolescents in grades 7-12.

Design A secondary data analysis of the Taiwan Global School-Based Student Health Survey's 2012-2016 data. The survey was anonymous, cross-sectional and nationwide.

Setting Taiwan high school students (grades 7-12). Participants The sample comprised 27525 students from junior high schools (grades 7-9), and senior high schools, comprehensive schools, vocational high schools and night schools (grades 10-12).

Main outcome measures The rate of ever having had sexual intercourse; the rates and factors of condom use at first and last sex.

Results The sexual intercourse rate in each school type (in ascending order) was junior high school (1.62\%), senior high school (4.14\%), comprehensive school (9.08\%), vocational high school (14.03\%) and night school (41.09\%). Condom use rate decreased from $57.07 \%$ $(95 \% \mathrm{Cl}=54.31 \%$ to $59.83 \%)$ at first sex to $25.72 \%$ $(95 \% \mathrm{Cl}=23.34 \%$ to $28.10 \%)$ at last $\operatorname{sex}(\mathrm{p}<0.0001)$. The condom use rate (in ascending order) was junior high school (first sex: $37.67 \%$, last sex: $19.76 \%$ ), night school (55.83\%, 22.62\%), vocational high school $(61.13 \%$, $25.78 \%)$, comprehensive school $(62.83 \%, 28.61 \%)$ and senior high school $(68.38 \%, 34.96 \%)$. Older age at sexual debut was associated with condom use at first and last sex, and having one sexual partner was associated with condom use at last sex, as revealed by logistic regression analysis.

Conclusions This study highlights an urgent need to instil a proper understanding of protected sex while adolescents are still in their formative years. Despite the low sexual intercourse rate (4.95\%), there is lower condom use at last sex than at first sex, which indicates that many sexually active adolescents are not practising protected sex, especially among junior high school students.

\section{INTRODUCTION}

Adolescent sexual behaviour is an important public health issue because of its association with the risk of contracting sexually transmitted
Strengths and limitations of this study

- The Taiwan Global School-Based Student Health Survey (GSHS) was cross-sectional and based on a large nationally representative sample.

- The survey was anonymous and therefore encouraged honest responses to sensitive questions (such as sexual behaviours) from participants.

- Cross-country comparisons of the rates of sexual intercourse and condom use among adolescents based on GSHS data should be cautious because of the following reasons:

- 'Sexual intercourse' was not defined explicitly; therefore, its exact meaning is open to interpretation by the participants and varies across cultures.

- Inadequate details about sexual behaviours and condom use, such as knowledge, attitude and frequency of the behaviours.

infections (STIs), unintended pregnancies, abortions, non-consensual encounters, co-use of illicit or dangerous substances, and subsequent psychological stress. ${ }^{1}$ Examples of high risk sexual behaviours include unprotected sexual intercourse, having sexual intercourse with multiple partners and men who have sex with men. ${ }^{12}$ In Taiwan, the number of newly reported cases of human immunodeficiency virus / acquired immunodeficiency syndrome (HIV/AIDS) increased from 2012 to $2016,{ }^{3}$ and about $29 \%$ of all new cases of HIV infection occurred in adolescents and young adults (aged 15-24 years) in 2016. The estimated incidence rate of HIV among adolescents aged 13-24 years was about 20 per 100000 population during this period. ${ }^{5}$ Condoms are the most effective method to protect against STIs. Condom use at last high-risk sex is one of the indicators of the Millennium Development Goals' monitoring that was established by the United Nations International Children's Emergency Fund to tackle STIs. ${ }^{6}$ In Taiwan, the sexual intercourse 
rates among adolescents aged $13-15$ years increased from $2.2 \%$ (male) and $1.8 \%$ (female) in 2006 to $5.4 \%$ (male) and $5.3 \%$ (female) in $2016 .{ }^{7}$ Furthermore, the contraception use rate among these adolescents increased from $56.2 \%$ (male) and $58.1 \%$ (female) in 2006 to $74.8 \%$ (male) and $77.7 \%$ (female) in $2016 .{ }^{7}$ However, the predominant form of contraception was not reported. More importantly, whether sexually active adolescents in Taiwan actually practise protected sex (ie, use condoms) remains unclear.

It has long been speculated that adolescent sexual behaviours are associated with school type and education programmes. ${ }^{8}$ In Taiwan, public education has been compulsory from primary school to junior high school since 1968. Since 2014, a full 12-year compulsory education programme has been implemented. Junior high school students in grade 9 take a high school entrance examination to continue their education, which is designed as a screening tool to assign students to various school programmes for grades 10-12. Students who aspire to receive higher education mostly attend regular senior high schools. Other educational opportunities include vocational high schools and comprehensive schools, which are workplace oriented and emphasise vocational skills. Night school curricula vary, ranging from vocational to academic courses. Therefore, in this study, we aimed to identify the sexual intercourse and condom use rates at first and last sex among Taiwanese adolescents across school types by using nationally representative data of grade 7-12 students. To our knowledge, this study is the first to examine the rate at which sexually active adolescents in Taiwan practise protected sex by using the data from a large nationally representative sample.

\section{METHODS}

We performed a secondary analysis of the 2012-2016 data of the Taiwan Global School-Based Student Health Survey (GSHS). The Taiwan GSHS is a nationwide cross-sectional study that surveys the health behaviours of grade 7-9 (aged 12-15 years) and grade 10-12 (aged 15-18 years) students every other year. $^{9}$

The survey was conducted by the Health Promotion Administration (HPA), Ministry of Health and Welfare of Taiwan, in collaboration with the United States Centers for Disease Control and Prevention. ${ }^{9}$ The Taiwan GSHS was translated into Chinese from the English version. The HPA first categorised schools into four geographical levels (ie, big city, small city, urban town and rural town) according to the population density of their locations. Next, the HPA used a two-step 'probability proportional to size' method at each geographical level for sampling, where the sample schools were drawn before the sample classes. ${ }^{9}$ They invited all students from the sample classes to participate. The survey was anonymous and no identifying information was collected. Additional details of the Taiwan GSHS's methodology are reported elsewhere. ${ }^{9}$
From 2012 to 2016, there were 29040 participants, and the response rates were $93.3 \%$ in $2012,89.6 \%$ in 2013 , $93.7 \%$ in 2014, $90.2 \%$ in 2015 and $92.6 \%$ in $2016 .{ }^{10}$ Junior high school students were surveyed in 2012, 2014 and 2016, and grade 10-12 students (including senior high school, comprehensive, vocational high school and night school students) were surveyed in 2013 and 2015. As illustrated in figure 1, this study included a total of 27525 students, after excluding those who did not indicate their gender $(n=62)$ and those who gave contradictory answers to the eight survey questions (online supplemental table S1, online supplemental material) about sexual behaviours $(\mathrm{n}=1453)$.

We selected three sections of questions from the GSHS (online supplemental table S2): demographics, substance use and sexual behaviours. The demographic variables included gender, age, degree of urbanisation, school type and grade. Regarding substance use, we looked into whether the participants were current smokers (defined as smoking cigarettes on at least 1 day during the past 30 days) $^{11}$ and current heavy alcohol users (defined as consuming $\geq 5$ drinks of alcoholic beverages per day in the past 30 days). ${ }^{12}$ The variables regarding sexual behaviours were ever having had sexual intercourse, number of sexual partners, condom use, and non-condom use at first sex and last sex. The outcome variables were ever having had sexual intercourse and condom use at first and last sex. However, since questionnaires of Taiwan GSHS did not explicitly define 'sexual intercourse', its exact meaning was open to interpretation by the subjects.

We used the $\mathrm{X}^{2}$ test to examine the association between the students' gender and the outcome variables (sexual intercourse and condom use at first and last sex) in each school type. We conducted McNemar's test to investigate the consistency of condom use at first and last sex for students who had one sexual partner and those who had more than one sexual partner. In univariate analysis, we used $\mathrm{X}^{2}$ test to examine the association between demographics, risk/protective factors and outcome variables (condom use at first and last sex). Regarding multivariate analysis, we used logistic regression with forward selection to locate important factors for condom use at first and last sex. The adjusted odds ratio (AOR) with 95\% CI shows the direction and magnitude of the association between demographics, risk/protective factors and outcome variables (condom use at first and last sex). We conducted all analyses using SAS V.9.4 (SAS Institute). The significance level was 0.05 .

\section{Patient and public involvement}

No patients were involved.

\section{RESULTS}

\section{Sexual intercourse rate by gender, school type and grade}

Overall, the sexual intercourse rate was $4.95 \%$ (figure 1). The ascending order of sexual intercourse rate by school type was junior high school (1.62\%), senior high school 


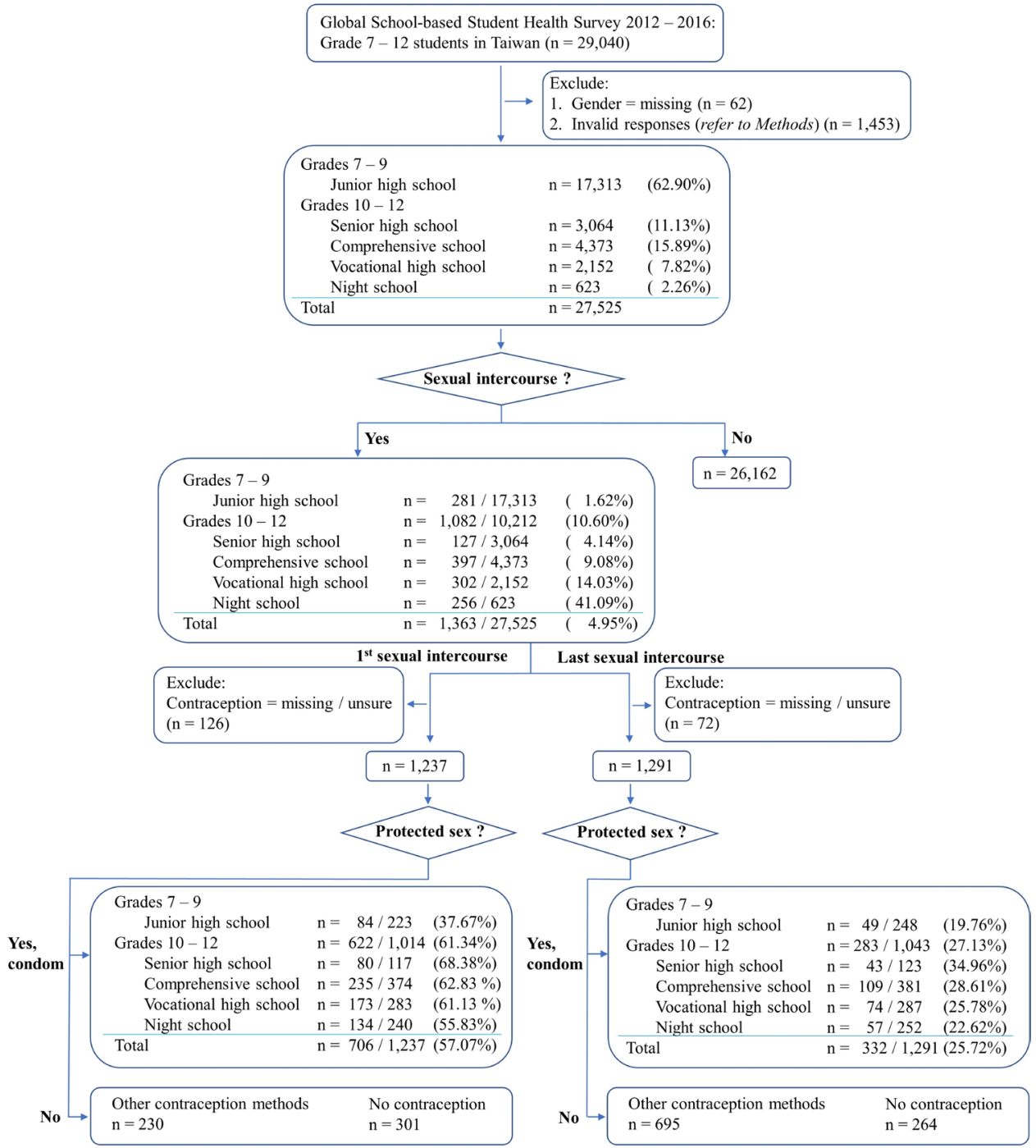

Figure 1 Flow chart of analysis illustrating the overall rates of ever having had sexual intercourse and condom use among adolescents in Taiwan from 2012 to 2016.

$(4.14 \%)$, comprehensive school $(9.08 \%)$, vocational high school $(14.03 \%)$ and night school $(41.09 \%)$.

In general, male students reported higher rates of sexual intercourse $(5.33 \%)$ than female students $(4.53 \%)$ (table 1). However, there was significant interaction between gender and school type $(p=0.0279)$, which indicated that the apparent difference in rate of sexual intercourse between both genders depended on different school types or vice versa. Therefore, the main effect of gender or that of school type on sexual intercourse was meaningless. When stratified by school type, male students in senior high school had a significantly higher rate of sexual intercourse $(4.92 \%)$ than female students $(3.4 \%) \quad(p=0.0352)$. There was a borderline difference in sexual intercourse rate among students at vocational high school between both genders $(p=0.0613)$. However, when the sexual intercourse rates were further stratified by grade and school type, the rates of sexual intercourse between both genders did not differ significantly (figure 2).

\section{Condom use at first and last sexual intercourse}

The overall condom use rate decreased from $57.07 \%$ at first sex to $25.72 \%$ at last sex (figure 1). The condom use rates were not statistically different between male and female adolescents at both first and last sex, with $\mathrm{p}=0.3164$ and $\mathrm{p}=0.9545$, respectively (table 1 ). In terms of school type, the ascending order of the condom use rate was junior high school (first sex: $37.67 \%$, last sex: $19.76 \%$ ), night school $(55.83 \%, 22.62 \%)$, vocational high school $(61.13 \%, 25.78 \%)$, comprehensive school (62.83\%, $28.61 \%)$ and senior high school (68.38\%, 34.96\%) (figure 1). Moreover, across all school types, condom use rates did not differ significantly between male and female students (table 1).

Univariate analysis showed that school type, age at first sexual intercourse, number of sexual partner(s), and being current smoker (in the past 30 days) were all significantly associated with condom use at first and last sex. We also noted that alcohol consumption was significantly associated with condom use at first $\operatorname{sex}(\mathrm{p}=0.0037)$ but not 
Table 1 Rates of ever having had sexual intercourse, condom use at first and last sex among adolescents in Taiwan from 2012 to 2016, by gender

\begin{tabular}{|c|c|c|c|}
\hline & Male (\%) & Female (\%) & $P$ value \\
\hline \multicolumn{4}{|l|}{ Grades 7-9 } \\
\hline \multicolumn{4}{|l|}{ Grades 10-12 } \\
\hline Senior high school & $74 / 1505$ (4.92) & $53 / 1559(3.4)$ & $0.0352 \dagger$ \\
\hline Vocational high school & 132/1048 (12.6) & 170/1104 (15.4) & 0.0613 \\
\hline Night school & $173 / 433(39.95)$ & $83 / 190(43.68)$ & 0.3836 \\
\hline \multicolumn{4}{|c|}{ Condom use at first sexual intercourse } \\
\hline Total & $384 / 688(55.81)$ & $322 / 549(58.65)$ & 0.3164 \\
\hline \multicolumn{4}{|l|}{ Grades 7-9 } \\
\hline Comprehensive school & $142 / 224(63.39)$ & $93 / 150(62.00)$ & 0.7847 \\
\hline Vocational high school & 72/121 (59.50) & 101/162 (62.35) & 0.6276 \\
\hline Night school & $89 / 160(55.63)$ & $45 / 80(56.25)$ & 0.9268 \\
\hline \multicolumn{4}{|c|}{ Condom use at last sexual intercourse } \\
\hline Total & $186 / 725$ (25.66) & $146 / 566(25.80)$ & 0.9545 \\
\hline \multicolumn{4}{|l|}{ Grades 7-9 } \\
\hline Junior high school & 26/133 (19.55) & 23/115 (20.00) & 0.9291 \\
\hline Grades $10-12$ & 160/592 (27.03) & $123 / 451(27.27)$ & 0.9296 \\
\hline
\end{tabular}

$P$ values were obtained from $X^{2}$ test.

${ }^{*}$ The interaction between gender and school type is statistically significant $(\mathrm{p}=0.0279)$ when tested with logistic regression (model: sexual intercourse $=\beta_{0}+\beta_{1}$ school type $+\beta_{2}$ gender $+\beta_{3}$ school type $\times$ gender). $\dagger P<0.05$.

at last sex $(\mathrm{p}=0.4314)$. Regarding number of sexual partner(s), the condom use rate at first and last sex among those with one sexual partner was statistically significantly higher than that of those with multiple sexual partners. At first sex, the condom use rates among those with one sexual partner and multiple sexual partners were $61.72 \%$ and $53.71 \% \quad(\mathrm{p}=0.0048)$, respectively. At last sex, the condom use rate among those with one sexual partner $(31.17 \%=202$ of 648$)$ was higher than that of those with multiple sexual partners $(20.34 \%=130$ of 639$)(\mathrm{p}<0.0001)$ (table 2).

In multivariate analysis of condom use at first sex, the following respondents were more likely to use condom at first sexual intercourse: those who first had sex at age 12-13 (AOR 4.74, 95\% CI=2.02 to 11.1), age 14-15 (AOR $10.6,95 \% \mathrm{CI}=4.72$ to 23.8 ) and age 16 years or above (AOR 18.3, 95\% CI=8.20 to 40.9) when compared with those who had sex at age 11 years or younger (table 2 ).
Regarding condom use at last sex, the multivariate analysis indicated that adolescents who had one sexual partner (AOR 1.60, 95\% CI=1.23 to 2.07) when compared with those who had more than one, and those who first had sex at age 16 years or above (AOR 2.00, 95\% CI=1.05 to 3.83 ) when compared with those who had sex at age 11 years or younger were more likely to use condom at last sex (table 2).

When investigating the decrease in the condom use rate, we observed that about two-thirds of adolescents who used condoms at first sex $(n=702)$ switched to using a non-condom contraceptive method (410 of 702, 58.4\%) or having no contraception (28 of 702, 4.0\%) at last sex. In contrast, those who did not use any contraception $(\mathrm{n}=293)$ at first sex remained not using any contraception (178 of $293,60.8 \%$ ) at last sex, and those who used noncondom contraception $(\mathrm{n}=212)$ at first sex mostly did not change their method of birth control (150 of 212, 70.7\%) 

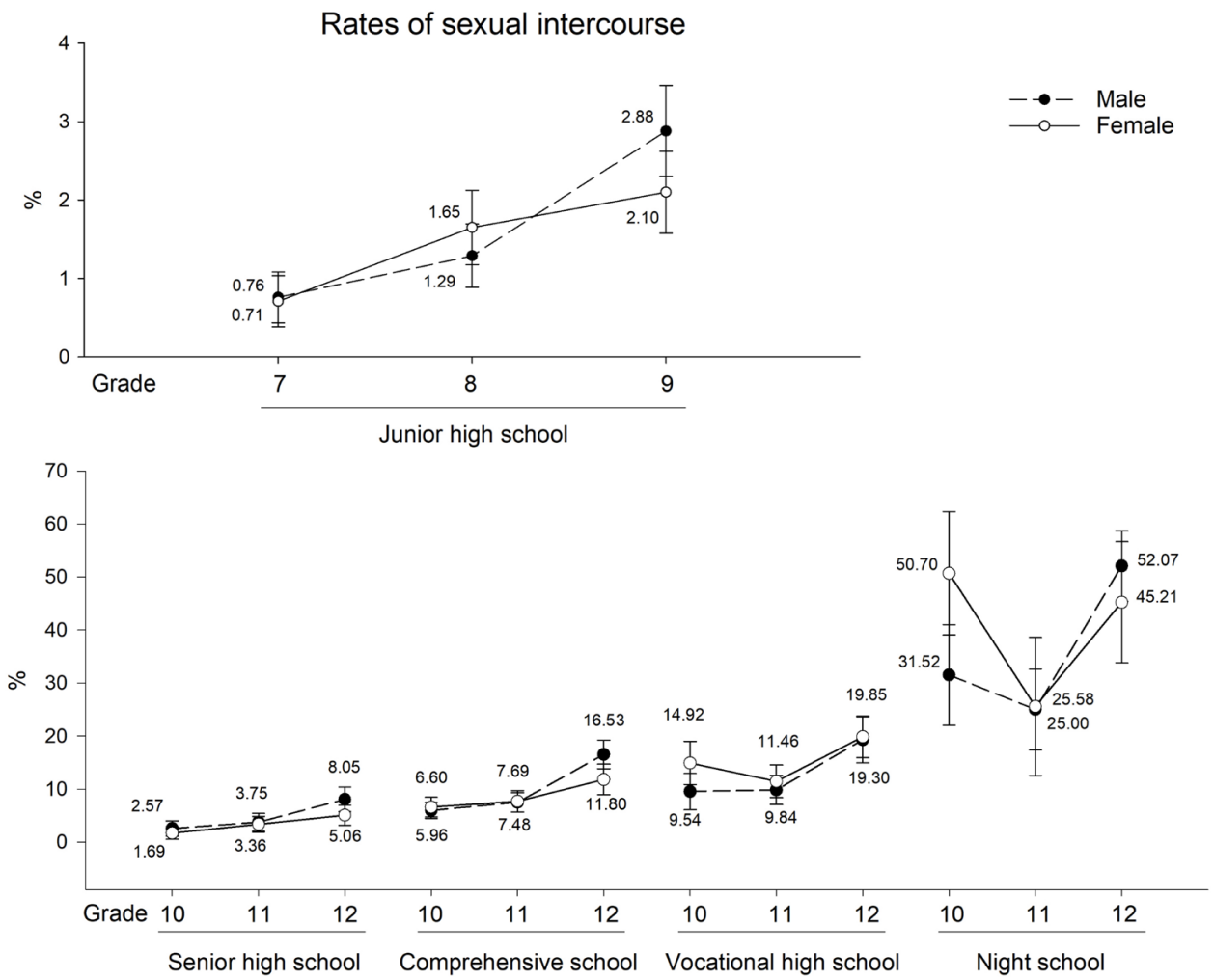

Figure 2 The rate of ever having had sexual intercourse of Taiwan adolescents from 2012 to 2016, by gender, school type and grade.

at last sex. Moreover, students with one sexual partner were more likely to use condoms both at first and last sex $(27.7 \%=168$ of 606$)$ than those with multiple sexual partners $(16.0 \%=96$ of 598$)(\mathrm{p}<0.0001) \quad($ table 3$)$.

\section{DISCUSSION}

\section{Sexual intercourse}

This nationwide cross-sectional study revealed that the overall rate of ever having had sexual intercourse among adolescents (grades 7-12) in Taiwan was $4.95 \%$ (5.33\% of males and $4.53 \%$ of females) during 2012-2016. Our rates are similar to several studies in other Asian countries and regions, such as South Korea (grades 7-12: $3.9 \%$ overall, opposite-sex intercourse; $5.5 \%$ of males, 2.2\% of females), ${ }^{13}$ Hong Kong (grades 7-12: $5.2 \%$ overall, $5.6 \%$ of males, $4.7 \%$ of females) ${ }^{14}$ and other Southeast Asian countries. ${ }^{15}$ On the other hand, our rates are considerably lower than those of developed Western countries. ${ }^{16}{ }^{17}$ For instance, in the USA, the sexual intercourse rate among grade 9-12 students is $41.2 \%$ (43.2\% of males and $39.2 \%$ of females).$^{17}$ Our rates are also lower than that of Australia, where it is geographically close to Southeast Asian countries but culturally similar to that of Great Britain and the USA. In Australia, the rates of anal or vaginal sex were $43.7 \%$ (male) and $48.5 \%$ (female) among year $10-12$ heterosexual students. ${ }^{18}$ It is possible that Confucian culture plays an important role in adolescent sexual behaviours in Asian countries, ${ }^{19}$ and this could potentially explain the discrepancy between our results and those obtained from Western countries.

More importantly, we observed that the rates of sexual intercourse varied greatly with different school types in Taiwan. Our analysis found that the sexual intercourse rate was lowest among junior high school students and that it generally increased with the grade. This was reasonable because more adolescents engaged in intimate sexual behaviours as they matured. Among the grade 10-12 students, night schools had the highest sexual intercourse rate $(41.09 \%)$, followed by vocational high school $(14.03 \%)$, comprehensive school $(9.08 \%)$ and senior high schools $(4.14 \%)$. We speculated that the highest rate of sexual intercourse among night school students $(41.09 \%)$ was probably because some of them were married. As previously hypothesised, ${ }^{8}$ the students' educational and career aspirations and the school type may be associated with their sexual behaviours. Our findings highlight that academically oriented schools (eg, senior high schools) tend to have lower sexual intercourse rates than those that place a heavier emphasis on vocational skill training (eg, comprehensive and vocational high schools).

After stratifying by school type and grade, the difference in rates of sexual intercourse between both genders was statistically non-significant, although male students seemed to have a higher overall rate $(5.33 \%)$ than female students $(4.53 \%)$. This finding is inconsistent with previous reports, where male adolescents generally 


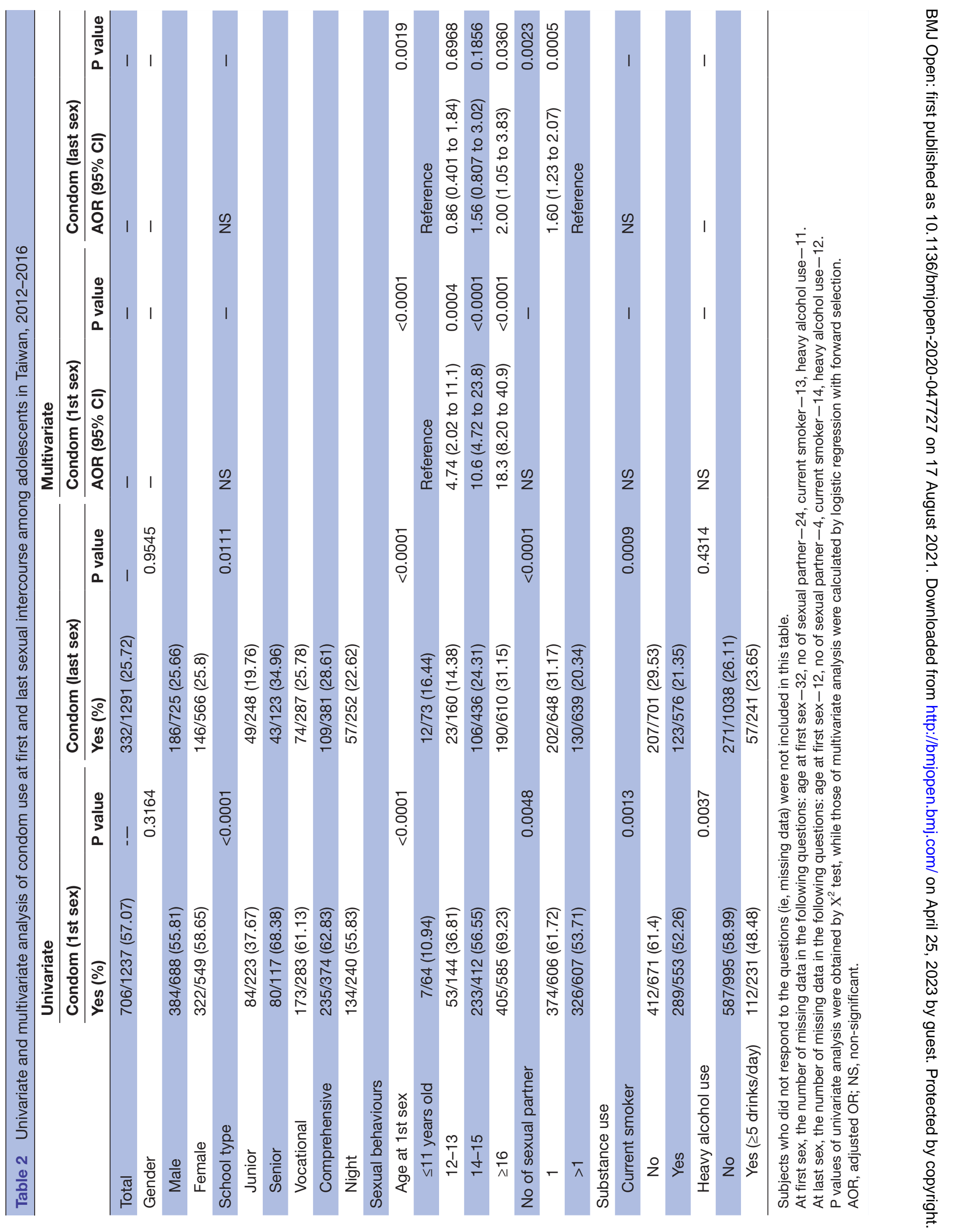


Table 3 Inconsistent use of condom at first and last sexual intercourse among adolescents in Taiwan from 2012 to 2016 , by number of sexual partner

\begin{tabular}{|c|c|c|c|c|c|}
\hline \multirow[b]{2}{*}{ First sexual intercourse } & \multirow[b]{2}{*}{ Total } & \multicolumn{4}{|c|}{ Last sexual intercourse } \\
\hline & & No contraception & Condom & Other methods & $P$ value \\
\hline \multicolumn{6}{|l|}{ Overall } \\
\hline No contraception & 293 & 178 & 25 & 90 & \\
\hline Condom & 702 & 28 & 264 & 410 & \\
\hline Total & 598 & 131 & 124 & 343 & $<0.0001$ \\
\hline No contraception & 158 & 93 & 15 & 50 & \\
\hline Condom & 326 & 18 & 96 & 212 & \\
\hline Other methods & 114 & 20 & 13 & 81 & \\
\hline Condom & 374 & 9 & 168 & 197 & \\
\hline Other methods & 98 & 15 & 14 & 69 & \\
\hline
\end{tabular}

$P$ values were obtained from McNemar's test.

The overall total $(1207)$ does not equal to the total $(598+606=1204)$ at the lower half of the table because subjects who did not respond to the questions about (1) the number of sexual partners, (2) the method of contraception use at first and last sexual intercourse were not included in McNemar's test.

had a higher sexual intercourse rate than female adolescents. ${ }^{19}$ In Confucian societies, where chastity is emphasised heavily, unmarried women engaging in sexual intercourse are highly stigmatised. ${ }^{19}$ Meanwhile, countries (eg, Taiwan) which were traditionally influenced by Confucianism are becoming westernised, possibly leading to increasing acceptance of premarital sexual behaviours among female adolescents. ${ }^{20}$ Taken together, this might potentially explain our finding that male and female adolescents did not differ significantly in rates of sexual intercourse after stratifying by school type and grade. Nevertheless, the degree to which Western culture involved in shaping Taiwan adolescents' sexual behaviours deserves further investigation.

\section{Condom use and implications}

Despite the low sexual intercourse rate, we found that the practice of protected sex (as indicated by the condom use rate) was not common among adolescents in Taiwan when compared with data obtained from Western ${ }^{21-23}$ and Southeast Asian countries. ${ }^{15}$ In this study, only a quarter of sexually experienced respondents had used a condom at last sex (male: $25.66 \%$, female: $25.80 \%$ ), which was approximately half of those who used condoms at first sex (male: $55.81 \%$, female: $60.47 \%$ ). In contrast, in Western countries such as the USA, the condom use rates at first sex among adolescents (age 15-17 years) are as high as $77 \%$ (male) and $74.6 \%$ (female) ${ }^{22}$ and the rates at last sex (age 13-19 years) are $61 \%$ (male) and $47 \%$ (female). ${ }^{21}$ In a study of nationwide surveys conducted in 33 countries from 2013 to 2014, developed Western countries, such as Great Britain, Denmark, Finland, France, Spain and
Switzerland, were found to typically have a condom use rate of over $40 \%$ at last sex among adolescents aged 14-16 years. ${ }^{23}$ Moreover, countries participating in the GSHS in the Southeast Asian and Western Pacific regions generally have a higher condom use rate (at last sex) than the rate $(25.72 \%)$ we obtained. ${ }^{15}$ For instance, the condom nonuse rates at last sex among GSHS participants aged 12-15 years in Nepal, Bangladesh, Thailand, Cambodia, Laos and Malaysia were estimated to be $52.6 \%$ (2015), $58.7 \%$ (2014), 33.8\% (2015), 56.2\% (2013), 46.7\% (2015) and $66.8 \%$ (2012), respectively. ${ }^{15}$ In Australia, despite being culturally Western but geographically proximal to Southeast Asia, the rates of condom use were considerably higher than our rates (In Australia, the condom use rates were $78.1 \%$ (male) and $77.5 \%$ (female) at first sex, and $65.1 \%$ (male) and $56.8 \%$ (female) at last sex). ${ }^{18}$ In summary, the above comparisons show that Taiwanese adolescents' condom use (both at first and last sex) is not as prevalent as in Western developed countries and other countries participating in the GSHS.

Sex education is critical to the practice of protected sex among adolescents. Although the use of contraception is covered in Taiwan sex education, the importance of safe sex and correct use of condom are not emphasised, and abstinence-only sex education remains the mainstream of sex education in Taiwan. ${ }^{24} 25$ In fact, condom vending machines and free condom programmes are not available in high schools in Taiwan. This is probably because providing condom vending machines for students might be mistakenly seen as encouraging students to have sex. These reasons might have contributed to the relative low rate of condom 
use among adolescents in Taiwan when compared with the rates in Western and GSHS-participating countries.

In addition to cross-country comparisons, we investigated how school type and the students' gender were associated with the condom use rate. Across school types, the condom use rate at first and last sex varied considerably. Notably, night schools had the highest sexual intercourse rate and the second lowest condom use rate at both first and last sex. Moreover, despite having the lowest sexual intercourse rate, sexually experienced junior high school students were the least likely to use condoms at first and last sex. Our multivariate analysis also revealed that adolescents who initiated sex at a younger age tended not to use condom. In fact, we found that age at first intercourse had the strongest association with condom use at first and last sex. This highlights the urgent need to instil a proper understanding of protected sex while adolescents are still in their formative years, especially in junior high schools.

Our study also showed that the students' gender did not seem to influence the condom use rate at first and last sex. It has long been assumed that gender equality plays an important role in condom use. ${ }^{23}$ Our study suggests that across all school types and grades, male and female adolescents in Taiwan are of equal standing in terms of condom use decisions, in contrast to studies conducted elsewhere. ${ }^{26}$

Apart from gender, substance use (ie, being current smoker and heavy alcohol use) was not found to be associated with condom use at first and last sex in our multivariate analysis (table 2). This contrasts with previous studies, which reported adolescents who engaged in risky sexual behaviours were more likely to use substances. ${ }^{27}$ There are many factors that are associated with both adolescent sexual behaviours and substance use, including mental disorders (especially attention-deficit/hyperactivity disorder), trauma, sexual abuse, etc. ${ }^{27-29}$ Therefore, whether the association between condom use and substance use exists among adolescents across different cultures and countries deserves further research.

The decrease in the condom use rate and increase in the non-condom contraception rate at last sex were possibly due to the adolescents being more concerned about potential pregnancy than the contraction of STIs, as speculated in previous studies. ${ }^{30}$ Our data support this hypothesis; most participants in our study who used condoms at first sex $(58.16 \%)$ changed to using noncondom contraceptive methods at last sex $(33.97 \%)$ (table 3). Our results also suggest that adolescents with one sexual partner were more likely to use condoms at last sex than those with multiple sexual partners (tables 2 and 3). This might be because those who stayed with a single sexual partner were more likely to understand the importance of protected sex and the risk associated with having multiple sexual partners than those who did not. Nevertheless, adolescents with multiple sexual partners who did not use condoms could potentially pose a huge public health risk in Taiwan, since having multiple sexual partners and sexual intercourse without using condoms are associated with the susceptibility to STIs. ${ }^{31}$

\section{Strengths and limitations}

A strength of this study is that our analysis was based on a large nationally representative sample. Moreover, no identifying information was collected in the survey to encourage honest response. The GSHS used in this study allows multinational comparisons between the sexual intercourse and condom use rates of Taiwan students and those of others. However, there are several limitations to our study.

First, sex is a sensitive issue in Taiwan; therefore, sexual behaviour data collected by the GSHS might be underreported and susceptible to recall bias. Second, since sexual intercourse was not defined explicitly, its exact meaning is open to interpretation by the participants. The GSHS did not ask about the gender of the participants' sexual partner(s). Thus, 'sexual intercourse' might be misinterpreted as other forms of sex ie, oral intercourse, or activities other than vaginal-penile penetration). Additionally, researchers using GSHS data regarding sexual intercourse from different countries should be very cautious because interpretation of sexual intercourse varies across cultures. Third, there was a large difference in the number of male and female participants in comprehensive and night schools. Since our data are a representative sample of grade 10-12 students, having more male students than female students in comprehensive and night schools implied a real male predominance in these school types. This male predominance can be partially explained by the fact that men tend to be the main source of family income in Taiwan. Moreover, having more male students than female students in comprehensive and night schools may introduce selection bias. However, we believe that the selection bias should be at minimum because most of our results (ie, the rates of sexual intercourse and condom use) were already stratified by school type. Fourth, the GSHS questionnaires did not contain sufficient details of knowledge, attitude and practice of condom use (and other sexual behaviours) among adolescents. Therefore, our comparison of the condom use rate at first and last sex is insufficient for drawing definite conclusions regarding condom use consistency. Additionally, respondents might use condoms and other contraceptive methods simultaneously, leading to biased responses. Fifth, the Taiwan GSHS did not have questions regarding the gender of the sexual partner(s) of the subjects. As a result, we were unable to analyse adolescent non-vaginal-penile sexual intercourse. To address these limitations, we recommend incorporating questions with a clear definition of sexual intercourse, questions about the frequency of condom use and gender of sexual partner(s) in future GSHS.

In conclusion, the finding of lower sexual intercourse and condom use rates at first and last sex than in many developed countries indicates that the practice of protected sex is not prevalent among Taiwanese adolescents. This is further supported by our findings that most adolescents who used condoms at first sex switched to noncondom contraception at last sex and that adolescents 
who initiated sex at younger age and with multiple sexual partners were less likely to use condoms.

Acknowledgements The authors would like to thank the study participants, staff from participating schools of Taiwan GSHS, and staff from Health Promotion Administration (HPA), Ministry of Health and Welfare of Taiwan who made this study possible.

Contributors WHSS designed the study, conducted data analysis and interpretation, and drafted the manuscript. P-RL and L-CS prepared the GSHS dataset, interpreted the data and revised the manuscript. All authors read and approved the manuscript in its present form.

Funding The authors have not declared a specific grant for this research from any funding agency in the public, commercial or not-for-profit sectors.

Competing interests None declared.

Patient consent for publication Not required.

Ethics approval The Institutional Review Board of the Chang Gung Medical Foundation approved this study and waived the requirement for informed consent for this anonymous survey (IRB number: 202001534B0).

Provenance and peer review Not commissioned; externally peer reviewed. Data availability statement № data are available.

Supplemental material This content has been supplied by the author(s). It has not been vetted by BMJ Publishing Group Limited (BMJ) and may not have been peer-reviewed. Any opinions or recommendations discussed are solely those of the author(s) and are not endorsed by BMJ. BMJ disclaims all liability and responsibility arising from any reliance placed on the content. Where the content includes any translated material, BMJ does not warrant the accuracy and reliability of the translations (including but not limited to local regulations, clinical guidelines, terminology, drug names and drug dosages), and is not responsible for any error and/or omissions arising from translation and adaptation or otherwise.

Open access This is an open access article distributed in accordance with the Creative Commons Attribution Non Commercial (CC BY-NC 4.0) license, which permits others to distribute, remix, adapt, build upon this work non-commercially, and license their derivative works on different terms, provided the original work is properly cited, appropriate credit is given, any changes made indicated, and the use is non-commercial. See: http://creativecommons.org/licenses/by-nc/4.0/.

\section{ORCID iDs}

Wing Hin Stanford Siu http://orcid.org/0000-0003-2876-219X

Pei-Ru Li http://orcid.org/0000-0001-7744-0555

Lai-Chu See http://orcid.org/0000-0002-1379-8969

\section{REFERENCES}

1 Chawla N, Sarkar S. Defining "high-risk sexual behavior" in the context of substance use. Journal of Psychosexual Health 2019:1:26-31.

2 Grubb LK, Adolescence CO, COMMITTEE ON ADOLESCENCE. Barrier protection use by adolescents during sexual activity. Pediatrics 2020;146:e2020007245.

3 Taiwan Centers for Disease Control. Statistics of HIV/AIDS. Available: https://www.cdc.gov.tw/En/Category/MPage/kt6yloEGURtMQubQ 3nQ7pA [Accessed 11 Sept 2020].

4 Centers for Disease Control, Ministry of Health and Welfare, R.O.C.(Taiwan). Cdc annual report, 2017. Available: https://www.cdc. gov.tw/En/InfectionReport/Info/BAkN3IDoa6hdrimSerBQyQ?infold= Otix8qZHC6IMEhPoOTMVDA [Accessed 18 June 2021].

5 Taiwan Centers for Disease Control. Epidemic trend and prevention effectiveness of HIV/AIDS. Available: https://www.cdc.gov.tw/En/ Professional/ProgramResultInfo/ppxd4Xu5zcYwcLHniXKk6w? programResultld=8XOWLPdD6JI5pNt9p0zrKQ [Accessed 6 Nov 2020].

6 Waage J, Banerji R, Campbell O, et al. The millennium development goals: a cross-sectoral analysis and principles for goal setting after 2015. The Lancet 2010;376:991-1023.

7 Health Promotion Administration, Ministry of Health and Welfare, Taiwan. Statistical yearbook of health promotion, 2016. Available: https://www.hpa.gov.tw/EngPages/Detail.aspx?nodeid=3850\&pid= 10649 [Accessed 6 Nov 2020].
8 Peterson AJ, Donze M, Allen E, et al. Effects of interventions addressing school environments or educational assets on adolescent sexual health: systematic review and meta-analysis. Int Perspect Sex Reprod Health 2018;44:111-31.

9 Health Promotion Administration, Ministry of Health and Welfare, Taiwan. Taiwan global school-based student health survey (GSHS). Available: https://www.hpa.gov.tw/Pages/Detail.aspx?nodeid=1077\& pid=6205 [Accessed 11 Sept 2020]

10 Health Promotion Administration, Ministry of Health and Welfare, Taiwan. Health promotion administration annual report. Available: https://www.hpa.gov.tw/EngPages/List.aspx?nodeid $=1070$ [Accessed 11 Sept 2020].

11 Jamal A, Gentzke A, Hu SS, et al. Tobacco use among middle and high school students - United States, 2011-2016. MMWR Morb Mortal Wkly Rep 2017;66:597-603.

12 Noel JK. Associations between alcohol policies and adolescent alcohol use: a pooled analysis of GSHS and ESPAD data. Alcohol Alcohol 2019;54:639-46.

$13 \mathrm{Kim} \mathrm{GH}$, Ahn HS, Kim HJ. Type of sexual intercourse experience and suicidal ideation, plans, and attempts among Youths: a crosssectional study in South Korea. BMC Public Health 2016;16:1229.

14 Wong WCW, Choi EPH, Holroyd E, et al. Impact of household composition and satisfaction with family life on self-reported sexual health outcomes of high-school students in Hong Kong. BMJ Sex Reprod Health 2020;46:184-91.

15 Smith L, Jackson SE, Vancampfort D, et al. Sexual behavior and suicide attempts among adolescents aged 12-15 years from 38 countries: a global perspective. Psychiatry Res 2020;287:112564

16 Scott RH, Wellings K, Lindberg L. Adolescent sexual activity, contraceptive use, and pregnancy in Britain and the U.S.: a Multidecade comparison. J Adolesc Health 2020;66:582-8.

17 Kann L, McManus T, Harris WA. Youth Risk Behavior Surveillance United States, 2015. MMWR Surveill Summ 2016;65:1-174.

18 Fisher CM, Kauer S, Mikolajczak G, et al. Prevalence rates of sexual behaviors, condom use, and contraception among Australian heterosexual adolescents. J Sex Med 2020;17:2313-21.

19 Zuo X, Lou C, Gao E, et al. Gender differences in adolescent premarital sexual permissiveness in three Asian cities: effects of gender-role attitudes. J Adolesc Health 2012;50:S18-25.

20 Cheng Y, Lou C, Gao E, et al. The relationship between external contact and unmarried adolescents' and young adults' traditional beliefs in three East Asian cities: a cross-sectional analysis. $J$ Adolesc Health 2012;50:S4-11.

21 Koumans EH, Welch R, Warner DL. 70. Differences in adolescent condom use trends by global region. Journal of Adolescent Health 2020;66:S36-7.

22 Holway GV, Brewster KL, Tillman KH. Condom use at first vaginal intercourse among adolescents and young adults in the United States, 2002-2017. J Adolesc Health 2020;67:606-8.

23 de Looze M, Madkour AS, Huijts T, et al. Country-level gender equality and adolescents' contraceptive use in Europe, Canada and Israel: findings from 33 countries. Perspect Sex Reprod Health 2019:51:43-53

24 Leung $\mathrm{H}$, Shek DTL, Leung E, et al. Development of contextuallyrelevant sexuality education: lessons from a comprehensive review of adolescent sexuality education across cultures. Int J Environ Res Public Health 2019;16:621.

25 Yang $\mathrm{H}-\mathrm{C}$. Teaching sexual matters in Taiwan: the analytical framework for popular culture and youth sexuality education. Asia Pacific Journal of Education 2014;34:49-64.

26 Vasilenko SA, Kreager DA, Lefkowitz ES. Gender, contraceptive attitudes, and condom use in adolescent romantic relationships: a Dyadic approach. J Res Adolesc 2015;25:51-62.

27 Khadr SN, Jones KG, Mann S, et al. Investigating the relationship between substance use and sexual behaviour in young people in Britain: findings from a national probability survey. BMJ Open 2016;6:e011961.

28 Chen M-H, Hsu J-W, Huang K-L, et al. Sexually transmitted infection among adolescents and young adults with attention-deficit/ hyperactivity disorder: a nationwide longitudinal study. J Am Acad Child Adolesc Psychiatry 2018;57:48-53.

29 Thompson R, Lewis T, Neilson EC, et al. Child maltreatment and risky sexual behavior. Child Maltreat 2017;22:69-78.

30 Samkange-Zeeb FN, Spallek L, Zeeb H. Awareness and knowledge of sexually transmitted diseases (STDs) among school-going adolescents in Europe: a systematic review of published literature. BMC Public Health 2011;11:727.

$31 \mathrm{Chu} \mathrm{J}-\mathrm{H}$, Huang $\mathrm{J}-\mathrm{H}$. A theory-based exploration of condomless anal intercourse intention among young men who have sex with men of different sexual roles in Taiwan. Arch Sex Behav 2018;47:2041-50. 